\title{
ALTERNATIVAS DE LA EVOLUCIÓN DEL BORDE OCCIDENTAL DE AMERICA DEL SUR DURANTE EL PROTEROZOICO
}

\author{
VÍCTOR A. RAMOS* \& GRACIELA I. VUJOVICK*
}

\begin{abstract}
ALTERNATIVAS DE LA EVOLUCIÓN DEL BORDE OCCIDENTAL DE AMERICA DEL SUR DURANTE EL PROTEROZOICO. The recent proposal of the S WEAT hypothesis has produced a series of alteraatives for the tectonic evolution and possible connection between the Laurentia and Gondwana Cratons. This paper summarizes the different alternatives and present the constraints based on a South American perspective of the tectonic evolution of the distinct cratonic blocks that are exposed in the southwestern margin of Gondwanaland. This paper compiled the present knowledge of the Middle Proterozoic metamorphic rocks known in different terranes or cratonic blocks such as Amazonia, Pampia, Arequipa Antpfalla and Patagonia. Based on this information a tentative tectonic evolution of the southwestern border of Gondwanaland is discussed focusing the main problems and incoherences of the proposed alternatives.
\end{abstract}

Keywords: Proterozoic Pangea, Western Gondwana, Pampia Craton, accretion, island ares.

\begin{abstract}
RESUMEN La reciente propuesta de la hipótesis SWEAT ha producido una serie de alternativas para la evolución tectónica y las posibles conexiones entre los Cratones de Laurentia y Gondwana. Este trabajo resume las diferentes alternativas y presenta las restricciones basadas en una perspectiva sudamericana de la evolución tectónica de los distintos bloques cratónicos, que están expuestos en el margen sudoccidental de Gondwana. Este trabajo compiló el conocimiento presente de las rocas metamórficas del Proterozoico Medio conocido en los terrenos o bloques cratónicos, tales como Amazonia, Pampia, Arequipa-Antofalla y Patagonia. Basado en esta información, se discute una evolución tectónica tentativa del borde sudoeste de Gondwana, enfocando los principales problemas e incoherencias de las alternativas propuestas.
\end{abstract}

Palabras clave: Pangea Proterozoica, Gondwana occidental, Craton de Pampia, acreción, arcos islándicos.

INTRODUCCIÓN El objetivo del presente trabajo es analizar las diferentes alternativas propuestas para el ensamble de las distintas unidades proterozoicas de la porción sur del continente sudamericano sobre la base de sus características tectónicas, magmáticas y metamórficas.

La hipótesis de que Laurentia se había amalgamado antes del Proterozoico Medio (Hoffman 1988), y que el urógeno de Grenville marginaba el Craton de Laurentia en su sector oriental, permitió inferir que todos los márgenes neoproterozoicos de Laurentia correspondían a márgenes pasivos (Fig. la).

La reciente propuesta de Moores (1991) presentó un nuevo ensamble de los grandes bloques cratónicos que integraban el Pangea Neoproterozoico, posteriormente reconocido como Supercontinente de Rodinia, por Hoffman (1992). Esto ha permitido individualizar una serie de márgenes pasivos conjugados como contraparte en diversos cratones continentales, lo que abrió una serie de expectativas con respecto a la ubicación del continente sudamericano en este contexto. A fin de sistematizar el análisis de las diferentes propuestas, se las ha dividido en función de sus características principales. Sobre esta base, se han identificado las siguientes alternativas:

América del Sur como un bloque único integrante del Gondwana occidental La Pangea neoproterozoica de Moores (1991) consideraba que el margen continental de la Orogenia Grenville de Laurentia era compartido con los remanentes de esta edad que se observan en la Placa Báltica. En su modelo, no intervienen los cratones de América del Sur.

La primer propuesta en la que se considera la parte cratónica de América del Sur corresponde a Dalziel (1991), quién interpretó la continuación del orógeno de Grenville en la Antártida Oriental. Si se acepta la continuidad entre
Antártida y América del Sur, se puede inferir que el continente que estaba en contacto con Laurentia era la parte cratónica sudamericana que integraba el Gondwana Occidental (Fig. Ib).

Si mantenía Antártida Oriental y Australia como contrapartes del margen de Laurentia Occidental, era necesario proponer una gran masa oceánica entre el Gondwana Occidental y el Oriental. Este océano fue denominado como el Océano de Mozambique, por Dalziel (1991). La propuesta de un cierre de este océano en el limitado tiempo disponible del Neoproterozoico despertó numerosas críticas de los investigadores de la geología de Mozambique (Stern et al. 1992).

América del Sur como una serie de cratones independientes de Laurentia La propuesta original de Dalziel (1991) fue mejorada al tener conciencia que los diferentes bloques cratónicos como Amazonia, África Occidental, el Congo y Kalahari, se han amalgamado en el Gondwana Occidental a través de una serie sucesiva de colisiones, tal como lo propusieran diversos autores para América del Sur (Ramos 1988, Unrug 1992, entre otros), para la orogenia Brasiliana, y en África (Kaz'min 1988 y trabajos posteriores) durante la orogenia Panafricana. Modelos de amalgamación, no tan movilistas, fueron propuestos por Brito Neves \& Cordani (1991), los que sin embargo reconocen que por lo menos los cratones principales han colisionado a través del consumo de corteza oceánica, logrando su ensamble a fines del Proterozoico.

Sobre esa base, Hoffman (1991) ubica yutaponiendo los segmentos del Orógeno de Grenville de Báltica y Amazonia con los de Laurentia (Fig. lc y Id). En Amazonia, interpreta como orógeno parte del Grenville al cinturón de Rondonia Sunsas, desarrollado entre los 1.200 y los $1.000 \mathrm{Ma}$. Al desconocer las edades Grenville que se observan en la parte

\footnotetext{
* Departamento de Ciencias Geológicas, Facultad de Ciencias Naturales y Exactas, Universidad de Buenos Aires, Pabellón II, Ciudad Universitaria, Nuñez, 1428 Buenos Aires, Argentina
} 


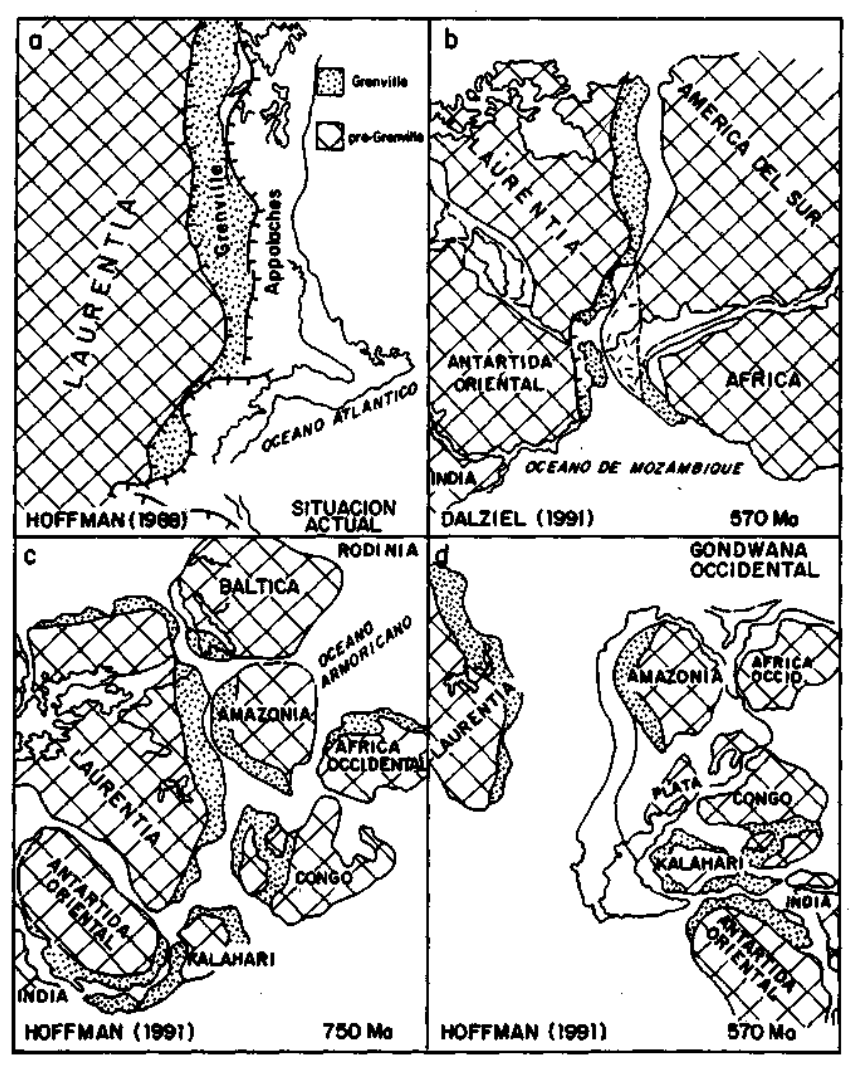

Figura 1 - a. Ubicación del Orógeno de Grenville en el margen occidental de Norte América (según Hoffman 1988); b. Propuesta del Pangea Proterozoico de Dalziel (1991), con ubicación del océano de Mozambique; c. Propuesta del Supercontinente Rodinia antes de su dispersión (Hoffman 1991); d. Desarrollo de lo Andes ancestrales como un orógeno del margen externo de Hoffman (1991) Figure 1 - a. Locations of the Grenville orogen in the western margin of North America (after Hoffman 1988); b. Proposal of Proterozoic Pangea by Dalziel (1991) with location of Mozambique Ocean; c. Proposal of the Rodinia Supercontinent before dispersa! (Hoffman 1991); d. Development of Ancestral Andes as an orogen of the external margin of Hoffman (1991)

noroeste de la Patagonia (Linares et al. 1985,1988), y en los sectores occidentales de las Sierras Pampeanas (Dalla Salda et al. 1992a, McDonough et al. 1993), interpreta a estos terrenos como de naturaleza oceánica, y por lo tanto no habrían intervenido en el ensamble de su Supercontinente de Rodinia, amalgamado a fines de Proterozoico Medio, principios del Superior. La falencia principal de la propuesta de Hoffman (1991) radica en interpretar toda la región cratónica al sur de Amazonia como un medio oceánico estructurado recién, durante la Orogenia Brasiliana.

Con posteridad Dalziel (1992a), teniendo conocimiento de las edades pre-Grenville de los cratones del Río de la Plata y Arequipa, junto con las edades cercanas a los 1,0 Ga de los macizos de Belén, integra el Orógeno Grenville con el de Báltica y Amazonia e incorpora en su paleogeografía a un océano Pampeano. Este océano tendría como islas los bloques cratónicos de pre-Grenville, los que posteriormente se aglutinarían durante la Orogenia Brasiliana (Fig. 2a y 2b). De esta forma, eliminaría los problemas suscitados por la creación del Océano de Mozambique, al considerar el Gondwana Occidental como una serie de bloques independientes. Una de las falencias de esta interpretación es no

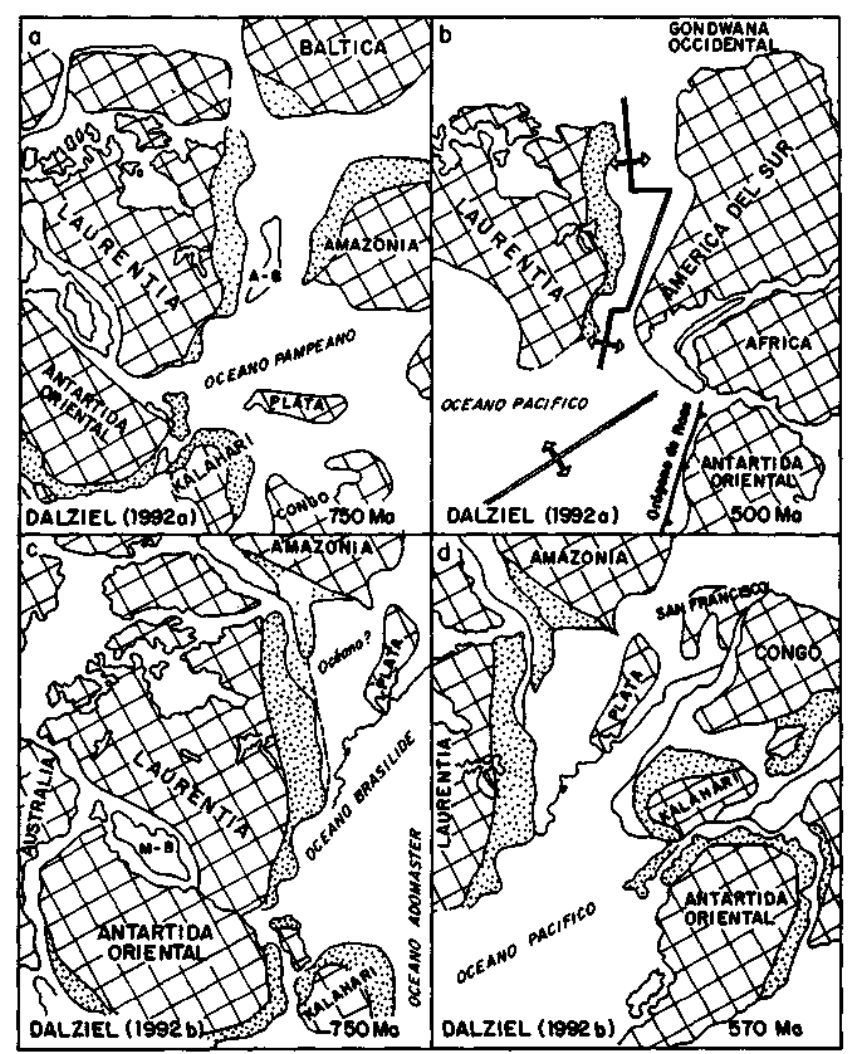

Figura 2 -a. Desarrollo del Océano Pampeano; b. Apertura del Océano lapetus y el Protopacífico, según Dalziel (1992a); c. Desarrollo del Océano Brasilide; d. Su cierre posterior, según Dalziel (1992b)

Figure 2 - (a) Development of Pampean Ocean and (b) aperture of lapetus Ocean and Protopacific (after Dalziel 1992a); (c) development of Brasilide Ocean and (d) its posterior closure (after Dalziel 1992b)

haber tenido en cuenta la edades Pre-Brasilianas existentes en diversos sectores del extremo sur de América del Sur.

Laurentia y América del Sur integradas en un único continente a fines de Proterozoico Medio Esta nueva propuesta considera que el Bloque Sudamericano y el de Laurentia estuvieron integrados, como resultado de la Orogenia de Grenville (Dalziel 1992b). En esta interpretacion, propone un gran océano denominado Brasilide (Fig. 2c), el que recién se habría cerrado uniendo el continente sudamericano con el africano durante la Orogenia Brasiliana - Panafricana (Fig. 2d). En este esquema, el Cratón de Kalahari pertenecería al Cratón de Antartida Oriental y estaría separado del resto de los cratones africanos por el océano de Adamastor (Hartnady 1991). El cierre de este océano durante la Orogenia Panafricana produjo el Pangea del Neoproterozoico Final.

El problema principal que tiene esta propuesta es que interpreta la ubicación relativa de los terrenos de Amazonia, San Francisco, Río de La Plata y parte de la Patagonia como fijos en una posición similar a la actual.

Dalla Salda et al. (1992a, b) proponen una interesante variante a la hipótesis de Dalziel, dado que interpretan que el Terreno Occidentalia, constituido por las Sierras Pampeana, la Puna, la Precordillera y parte del Macizo de Somuncura, eran parte del continente de Laurentia (Dalla Salda et al. 1992a). En esta propuesta, Laurentia habría 
colisionado con América del Sur durante la Orogenia Famatiniana - Tacónica en el Ordovícico Medio. Laurentia se habría separado a través de un sistema de rifting a partir del Ordovícico Medio-Superior. En esta alternativa, Precordillera sería un margen pasivo adyacente al de Ouachitas en Estados Unidos. Un rift se habría desarrollado entre el Silúrico y el Devónico para separar Laurentia del resto de Gondwana (Fig. 3a).

Sudamérica integrada por bloques independientes con una distribución paleogeográfica eventualmente diferente a la actual y amalgamada durante la Orogenia Brasiliana Esta alternativa es la que proponen los autores, interpretando que es la que mejor se ajusta a los datos geológicos, paleogeográficos y paleo-

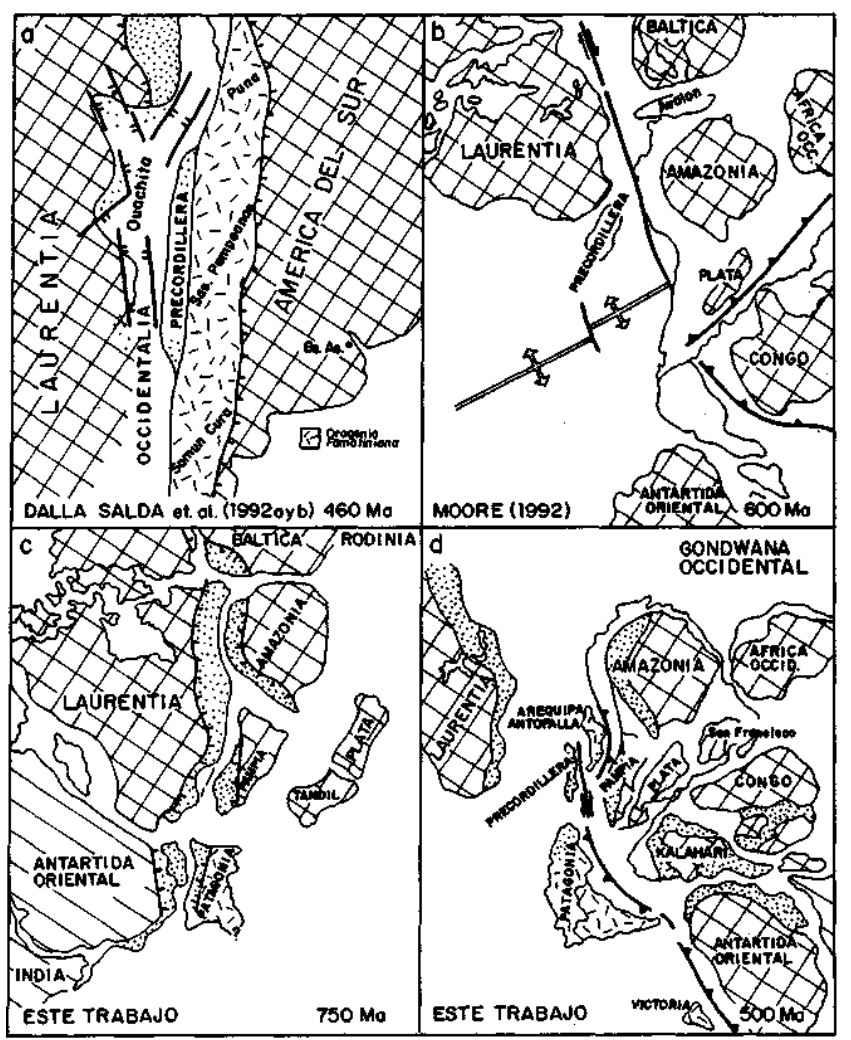

Figura 3-a. Paleogeografia durante la colisión famatiniana y desarrollo de rifts en el sector occidental de la Precordillera, según Dalla Salda et al. (7992 a y b); b. Propuesta de la conferencia de terrenos circumpacificos, según Moore (1992); c. Paleogeografía del Supercontinente de Rodinia propuesta en este trabajo. Nótese que la ubicación relativa de los diferentes terrenos es tentativa y que no se indica la posición de los terrenos africanos por la incertidumbre de su ubicación; d. Acrecían de diferentes terrenos durante el Paleozoico al margen del Gondwana Occidental La ubicación relativa de los terrenos africanos con respecto a los de América del Sur es posible debido a la amalgamación panafricana-brasiliana

Figure 3 - a. Paleogeography during the famatinian colusión with rifts development in western Precordillera (after Dalla Salda et al. 1992a, b); b. Proposal of the Circumpacific terrane conference (after Moore 1992); c. Paleogeography of Rodinia Supercontinent proposed here. Note that the relative location of the different terranes is tentative and the position of the African terranes is not shown due to uncertainty of their location; $\mathbf{d}$. Accretion of different terranes to Western Gondwana during the Paleozoic The relative location of the African terranes with respect to those of South America is possible due to the panafrican-brasilian amalgamation magnéticos de América del Sur. En forma previa a la presentación del modelo, se describirán las características más salientes de cada uno de los terrenos involucrados.

Precordillera Uno de los rasgos geológicos más salientes de la Precordillera es el desarrollo de una plataforma calcárea, interpretada por diversos autores como un margen pasivo eopaleozoico con un ordenamiento de facies más profundas, hasta oceánicas hacia el oeste (González Bonorino 1973,1975, Spalletti et al 1989). La Precordillera ha sido interpretada como un terreno independiente con afinidades apalachianas por Ramos et al (1986).

Los estudios de subsidencia de los depósitos de plataforma en la Precordillera de San Juan y Mendoza (Bond et al 1984, González Bonorino \& González Bonorino 1991) permiten establecer el pasaje de una subsidencia tectónica a una térmica, marcando el inicio del desarrollo de un margen pasivo entre los 575 y 560 Ma. La evolución geológica y las afinidades paleontológicas de la Precordillera con la Plataforma Carbonática de los Apalaches, indican que ambas se podrían haber formado a partir de un rifting iniciado aproximadamente a los 739 Ma (Rankin et al 1989). El mismo habría pasado de una subsidencia tectónica a una térmica alrededor de los 575 Ma (Fig. 4). Ambas plataformas calcáreas comparten una fauna de trilobites ollenélidos, sólo conocidos en Norte América y en la Precordillera de San Juan. Además, esta historia común de subsidencia se interrumpe en el Silúrico, donde aparentemente ambas plataformas han tenido una historia diferente. La Plataforma de la Precordillera inicia en este momento una aceleración de la subsidencia, la que es interpretada como relacionada al inicio de una carga tectónica sobre el sector oriental (Fig. 4).

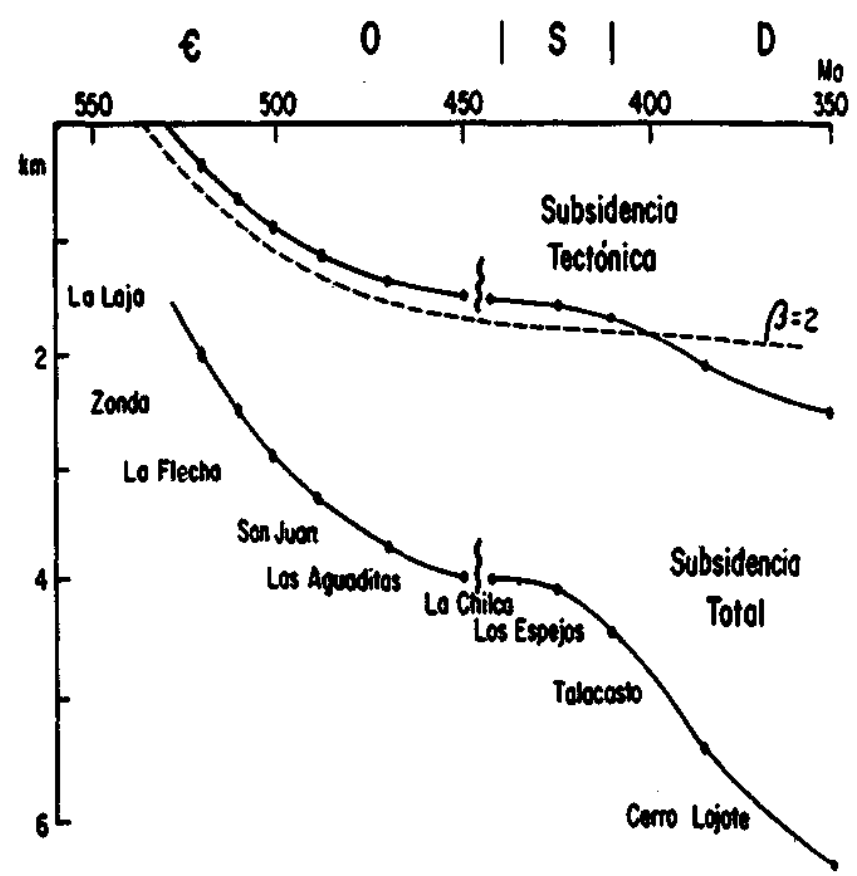

Figura 4 - Curva de subsidencia de la plataforma carbonática de la Precordillera, mostrando la transición entre la subsidencia térmica del margen pasivo, y la reactivación de la subsidencia por carga tectónica basado en González Bonorino \& González Bonorino 1991)

Figure 4 - Subsidence curve of the carbonate platform of Precordillera showing the transition between the thermal subsidence of the passive margin and the reactivation of the subsidence by tectonic loading (based on González Bonorino \& González Bonorino 1991) 
El basamento de la Precordillera sólo era conocido a través de los xenolitos traídos a la superficie por las rocas volcánicas terciarias (Leveratto 1968). Sólo recientemente se ha podido datar la edad de estos xenolitos y por ende del basamento de la Precordillera. Abbruzzi et al (1993) han obtenido sobre xenolitos silíceos del cerro Blanco de Zonda edades U-Pb de $1.188 \pm 122 \mathrm{Ma}$, en circones que han experimentado una pérdida de plomo durante el evento magmático mioceno. Las edades obtenidas, sobre la base de las relaciones isotópicas de $\mathrm{Pb}$, indicarían una evolución común de estos xenolitos que se remontaría a los $1.570 \mathrm{Ma}$.

El estudio de la población de circones en sedimentos del Paleozoico Inferior de la Precordillera, arrojó edades para la cristalización de los mismos de $1.100 \mathrm{Ma}$, mientras que otros redondeados indicaban edades mayores a 1.800 Ma (Loske 1992). Estos datos son coherentes con una procedencia de áreas aflorantes con Basamento de Grenville en las inmediaciones de la Precordillera.

Dado que la Precordillera se separó del resto de Laurentia a partir de los 575-560 Ma, y las primeras evidencias de amalgamación al terreno Pampia se constantan a partir del Ordovícico Superior - Silúrico, se interpreta que ésta fue un margen pasivo durante gran parte del Cámbrico. En algún momento del Ordovícico, ese margen se habría activado, como es constatado por las rocas magmáticas de esa edad emplazadas en la Precordillera. Sobre esta base, Moore (1992) presentó el ensayo paleogeográfico que se ilustra en la figura $3 \mathrm{~b}$.

Pampia Bajo esta denominación, Ramos \& Vujovich (1993) han propuesto agrupar el basamento de la región central de Argentina, que sólo parcialmente coincide con la expresión morfológica actual de las Sierras Pampeanas. Este basamento se extendería desde el límite con Paraguay, a través del arco Pampeano hacia el sur, hasta el Río Colorado. Hacia el este, estaría limitado con el Cratón del Río de La Plata, cuya sutura está parcialmente expuesta en el margen oriental de las sierras de Córdoba, marginada por la Faja Pampeana Oriental. Hacia el oeste, limita con la Precordillera en los sectores central y sur, mientras que en el noroeste está separado por la faja de Puncoviscana del terreno de Antofalla - Arequipa.

El sector oriental del Terreno Pampia, expuesto en las Sierras Pampeanas orientales, corresponde a un arco magmático desarrollado entre los 730 y 540 Ma (Ramos 1988). Está representado por gabros, granodioritas, tonalitas y granitos asociados a rocas metamórficas de mediano a alto grado, generadas a profundidades entre 7,1 y 7,4 kbs (Gordillo 1984). Estos cuerpos son atravesados discordantemente por diques graníticos y riolíticos, y en parte sobreyacidos por plateaus riolíticos en las sierras de Santiago del Estero y norte de Córdoba. Uno de estos diques arrojó una edad de $494 \pm 11$ Ma, mediante una isócrona $\mathrm{Rb}-\mathrm{Sr}$ con siete puntos de control (Rápela et al 1991). Esto indicaría un fuerte levantamiento de varios kilómetros del sector oriental del terreno Pampia a fines del Proterozoico o inicios del Cámbrico, como resultado del Ciclo Orogénico Brasiliano.

En este trabajo, se reconoce que una parte importante de las Sierras Pampeanas Occidentales, y posiblemente su extensión hacia el sur, tienen rocas metamórficas, plutónicas y metavolcánicas datadas entre 1.100 y $1.050 \mathrm{Ma}(\mathrm{U}-\mathrm{Pb}$ en circones Igneos y metamórficos) de una orogenia de Grenville (McDonough et al. 1993, Ramos et al. 1993) (Fig. 3c). Estas rocas pertenecen a asociaciones de arcos volcánicos islándicos, como se infiere por sus características geoquímicas (Vujovich et al. 1991, 1992). Estos arcos fueron acrecionados durante este evento al Cratón de Pampia. Estas rocas están expuestas en las sierras de
Pie de Palo y La Huerta, y posiblemente se continúen en las Sierras de Valle Fértil, Maz y Umango (Vujovich 1992,1993).

El terreno Pampia estuvo orlado en su margen occidental por una serie de depósitos turbidíticos del Proterozoico Superior a Cámbrico Inferior (Omarini 1983), que corresponden al prisma de acreción de la faja Punco viscana. Estos depósitos atestiguan el desarrollo de un sistema de rifting en el margen occidental de Pampia. Este rifting llegó a formar un verdadero margen pasivo (Jezek et al. 1985), con desarrollo de corteza oceánica y magmatismo de intraplaca oceánica (basaltos almohadillados del tipo IOB, según Coira et al. 1990). Este margen fue activado a finales del Proterozoico, como lo atestiguan los intrusivos de Santa Victoria, Tastil y Cachi, asociados a subducción del margen occidental de este terreno (Galliski et al. 1990, Schón \& Miller 1990, Grissom et al. 1991). La Orogenia Pampeana (Aceñola \& Toselli 1976) fue el resultado de la colisión del Bloque de Arequipa-Antofalla contra el Terreno Pampia, en el Cámbrico Medio (Aceñolaza 1982).

La historia paleozoica inferior del Terreno Pampia abarca casi exclusivamente su sector occidental. En éste, se distinguen intrusivos relacionados a subducción que alcanzaron su climax en el Ordovícico Medio-Superior, y que culminaron con granitos post-colisionales desde el Ordovícico más alto hasta el Carbónico Inferior inclusive (Rápelas al. 1992).

Patagónia Los estudios geocronológicos de Linares et al. $(1985,1988)$ han permitido constatar la presencia de rocas precámbricas en el basamento metamórfico del sector occidental del Macizo Nordpatagónico. Si bien estas edades confirman parcialmente el desarrollo del Urógeno de Grenville en este sector, la falta de asomos de basamento metamórfico impiden establecer su continuidad más al sur. Sin embargo, ya Cingolani \& Várela (1976) habían encontrado edades entre 980 y $1.100 \mathrm{Ma}$ en el único afloramiento de basamento de las Islas Malvinas en Cabo Belgrano.

No se conoce fehacientemente la edad del basamento de Patagonia, aunque es probable que parte del mismo podría tener edades pre-brasilianas.

Otros terrenos proterozoicos Aunque aún no se cuenta con dataciones precámbricas fehacientes en el terreno Chilenia (Ramos et al. 1984), las características geoquímicas del magmatismo posterior permitirían inferir la existencia de un importante sustrato siálico de posible edad precámbrica.

El Terreno de Antofalla, que comprende rocas metamórficas de mediano grado, tampoco cuenta con edades precámbricas, aunque está intruído por granitos cámbricos en Archibarca (Palma et al. 1986). Además, la continuidad de las anomalías de Bouguer positivas que desairóla (Gótze et al. 1987) permite inferir su posible continuidad con los Bloques de Belén y Arequipa. Estos últimos cuentam con edades absolutas correspondientes a la Orogenia de Grenville (Damm et al. 1990). Se ha extendido su desarrollo en subsuelo por debajo del Altiplano Boliviano en la figura 5 sobre la base de las dataciones de $1.050 \pm 100$ Ma obtinidas por $\mathrm{Rb} / \mathrm{Sr}$ en granitos gneissicos en testigos de perforaciones a 2.744 m b.b.p. (Lehmann 1978).

Hipótesis propuesta A fin de reconstruir la paleogeografía proterozoica de este sector del Gondwana, es necesario tener presente que al oeste del Cratón del Rio de la Plata existe una serie de cratones o terrenos alóctonos a este cratón, como los de Arequipa - Antofalla, Pampia, Patagonia y Chilenia, con edades precambricas.

Entre el Cratón de Amazonas y los cratones colindantes (Fig. 5), hacia el oeste y el sur, existió una faja plegada 


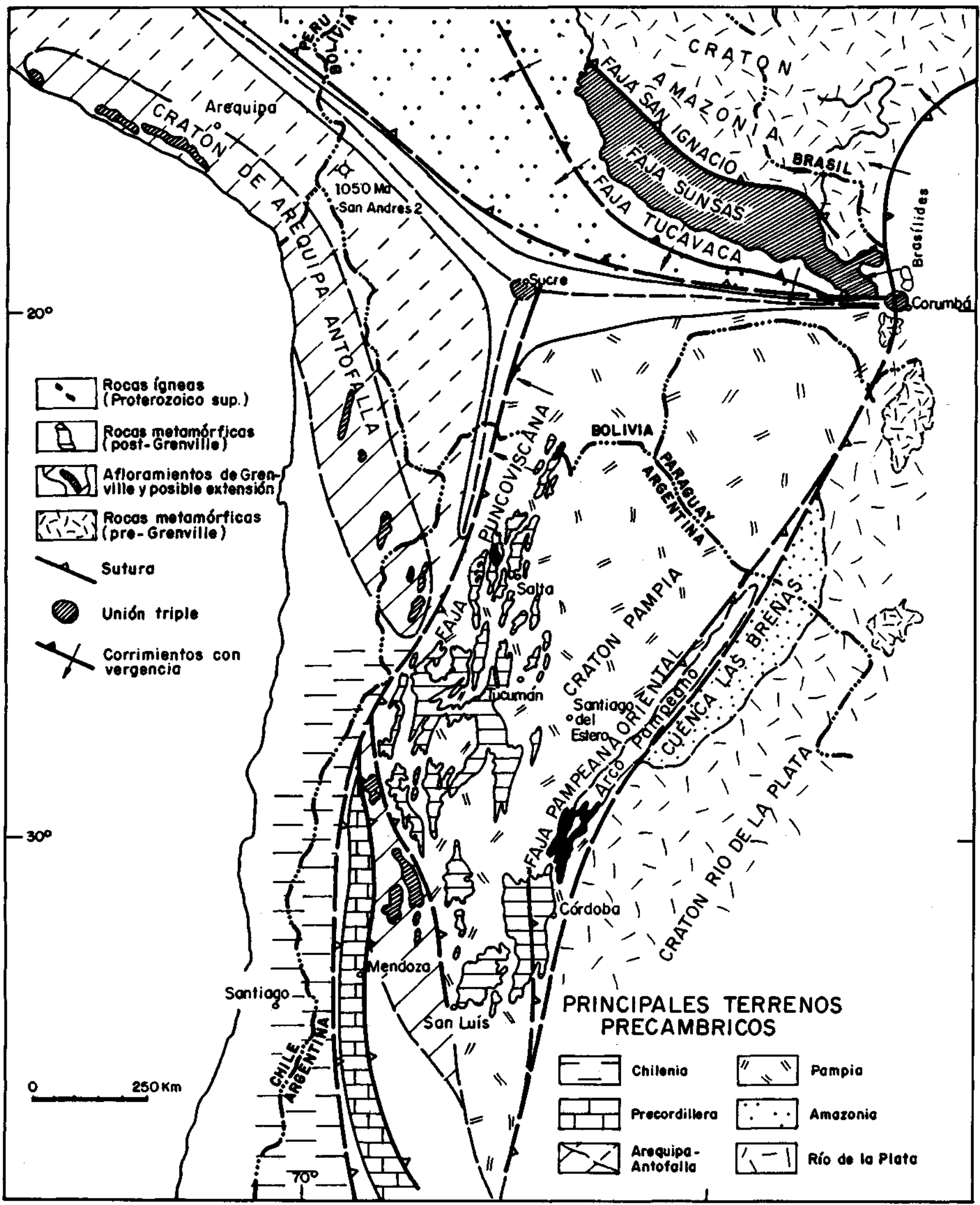

Figura 5- Esquema paleogeográfico mostrando los diferentes cratones proterozoicos distinguidos en el margen sudoeste del Gondwana (basado en Ramos 1988, Litherland et al. 1959 y observaciones propias de los autores)

Figure 5 - Paleogeographic scheme showing the different proterozoic cratons of the southwestem margin of Gondwana (based on Ramos 1988, Litherland et al. 1989 and the authors own observations) 
y corrida como la de Tucavaca, con vergencia hacia el noroeste y un posible margen activo alrededor de los $600 \mathrm{Ma}$, correspondiente al Ciclo Brasiliano (Litherland et al. 1989). Su orientación relativa con respecto al Cratón de Arequipa-Antofalla permitiría inferir que la Faja de Sunsas, de edad Grenville, habría estado sometida principalementea esfuerzos de cizalla levógira durante el Ciclo Brasiliano, lo que explicaría el escaso magmatismo de este margen. Esta faja plegada y corrida se desarrollaría al occidente déla Sinclinal de Tucavaca, y se conoce exclusivamente por datos sísmicos del subsuelo. Entre el Cratón de Amazonas y el Terreno Pampia, se continúa el Cinturón de Tucavaca, que está en continuidad con los brasilides del Cinturón Paraguai-Araguaia (Almeida et al. 1981). Esto permitiría deñnir dos puntos de unión triple, uno al este, el de Corumbá (Litherland et al. 1985), entre Amazonia Pampia-Río de La Plata, y el de Sucre, al oeste (Suárez Sónico \& López Paulsen 1992), entre Amazonia-Pampia-Arequipa. Aparentemente ambos han sido TTT, pero la colisión y cierre de ambos han sido diacrónicos, con el de Corumbá levemente más antiguo que el de Sucre. Ambas uniones triples habrían controlado con posterioridad el desarrollo de las cuencas eopaleozoicas del ciclo tacsariano (Cambro-Ordovícico de Bolivia), así como la deformación oclóyica y el desarrollo de la cuenca silurodevónica.

Si se acepta que la posición actual de estas tres placas proterozoicas ha sido obtenida durante el Ciclo Brasiliano-
Pampeano, a fines del Proterozoico o el Cámbrico Inferior, la posición relativa de estos bloques durante la Orogenia de Grenville es incierta. La figura $3 c$ muestra en forma tentativa las relaciones de estos terrenos con Laurentia ante la incertidumbre de su posición paleomagnética.

Los terrenos de Precordillera, Chilenia y Patagonia se habrían acrecionado al margen sudoccidental del Gondwana durante el Paleozoico (Ramos 1988).

CONCLUSIONES Si bien no hay acuerdo y quedan aún numerosos problemas paleogeográficos a resolver, la presentación de las diferentes alternativas en esquemas paleogeográficos con un lenguaje común permite focalizar las diferencias y las similitudes de los distintos modelos. Para comprender las implicancias de cada uno de ellos, es necesario primeramente tener en cuenta si los distintos autores se acogen a una tectónica movilista o fijista para la aglutinación y desarrollo de los núcleos cratónicos de Sudamérica. Si se acepta que los diferentes terrenos que componen Sudamérica han sido recién aglutinados durante el Proterozoico Superior y el Paleozoico Inferior, se hace evidente que la paleogeografia para el supercontinente de Rodinia, durante el episodio Grenville, o para el Supercontinente de Gondwana, durante el episodio Brasiliano, es más compleja que cualquiera de los modelos analizados.

\section{REFERENCIAS BIBLIOGRÁFICAS}

ABBRUZZI, J.M.L; KAY, S.M.; BICKFORD, E. 1993. Implications for the nature of the Precordilleran basement from the geochemistry and age of Precambrian xenoliths in Miocene volcanic rocks, San Juan province. In: CONGR. GEOL. ARGENT., 12. San Juan, 1993. Actas... San Juan, Asoc. Geol. Argent. v. 3, p. 331-339.

ACENOLA, G.F. 1982. El Sistema Ordovícico en Sudamérica. Ac. Geol. Lilloana, 16(1):77-109.

ACENOLAZA, F.G. \& TOSELLI, A. 1976. Consideraciones estrati gráficas sobre el Paleozoico Inferior del Noroeste Argentino. In: CONGR. LATINOAM. GEOL., 2. Caracas, 1976. Mem. Caracas, Dirección de Geología, v. 2, p. 755-763.

ALMEIDA, F.F.M.; HASUI, Y.; BRITO NEVES, B.B.; FUCK, R.A. 1981. Brazilian structural provinces: an introduction. Earth-Sci. Rev., 17:1-29.

BOND, G.C.; NICKELSON, P.A.; KOMINZ, M.A. 1984. Breakup of a supercontinent between 625 Ma and 55 Ma: new evidence and implications for continental histories. Earth Planet. Sci. Lett., 70:325-345.

BRITO NEVES, B.B. \& CORDANI, U.G. 1991. Tectonic evolution of South America during the Late Proterozoic. Precambrian Res., 53:23-40.

CINGOLANI, C. \& VÁRELA, R. 1976. Investigaciones geológicas y geocronológicas en el extremo sur de la Isla Gran Malvina, sector cabo Belgrano (Cabo Meredith), Islas Malvinas. In: CONGR. GEOL. ARGENT., 4. Bahía Blanca, 1975. Acias... Bahía Blanca, Asoc. Geol. Argent. v. 1, p. 457-474.

COIRA, B.; MANCA, M.; CHAILE, W. 1990. Registros volcánicos en la Formación Puncoviscana. In: ACENNOLAZA, F. G.; MILLER, H.; TOSELLI, AJ. eds. El Ciclo Pampeano en el Noroeste Argentino. Tucumán, Universidad Nacional de Tucumán. p. 53-60. (Serie Correlación Geológica 4).

DALZIEL, I.W.D. 1991. Pacific margins of Laurentia and East-AntarcticaAustralia as a conjúgate rift pair: evidence and implications for an eocambrian supercontinents. Ann. Rev. Earth Planet. Sci., 20:501-526.

DALZIEL, I.W.D. 1992b. On the organization of american plates in the Neoproterozoic and the breakout of Laurentia. GSA Today, 2(11):240-241.

DALLA SALDA, L.; DALZIEL, I.W.; CINGOLANI, C.; VÁRELA, R. 1992a. Did the Taconic Appalachians continué into southern South America? Geology, 20:1059-1062.

DALLA SALDA, L.; CINGOLANI, C.; VÁRELA, R. 1992b. The Early Paleozoic Famatinian belt of the Andes in southwestern South America: result of Laurentia-Gondwana colusión? Geology, 20:617-620.

DAMM, K.W.; PICHOWIAK, S; HARMON, R.S.; TODT, W.; KELLEY,
S.; OMARINI, R.; NIEMEYER, H. 1990. Pre-Mesozoic evolution of the central Andes; the basement revisited. In: KAY, S.M. \& RÁPELA, C.W. eds. Plutonism from Antárctica toAlaska. Geol. Soc. Am., Spec. Paper, 241:101-126.

GALLISKI, M.A.; TOSELLI, AJ.; SAAVEDRA, J. 1990. Petrology and geochemistry of the Cachi high-alumina trondhjemites, northwestem Argentina. Geol. Soc. Am.. Spec. Paper, 241:91-100.

GONZALEZ BONORINO, G. 1973. Sedimeniology and Paleogeography ofa Devonian Turbidite Basin in Argentina. Canadá. 137 p. (Master Se, Thesis, McMaster University)

GONZÁLEZ BONORINO, G. 1975. Sedimentología de la Formación Punta Negra y algunas consideraciones sobre la geología de la región de la Precordillera de San Juan y Mendoza. Asoc. Geol. Argent. Rev., 30(3):223-246.

GONZÁLEZ BONORINO, G. \& GONZÁLEZ BONORINO, F. 1991 Precordillera de Cuyo y Cordillera Frontal en el Paleozoico temprano: terrenos bajo sospecha de ser autóctonos. Rev. Geol. Chile, 18(2):97-108.

GORDILLO, C.E. 1984. Migmatitas Cordietíticas de la Sierra de Córdoba; Condiciones Físicas de Migmatización. Córdoba, Acad. Nac. Ciencias Córdoba, p. 1-37. (Mise. 68).

GOTZE, H.J.; CHONG DÍAZ, G.; LAHMEYER, A.; OMARINI, R.H.; SALFTTY, J.A.; SCHMIDT, S.; STRUNK, S.; VIRAMONTE, J.G. 1987. The gravity field and its relation to the Andean volcanism between $20^{\circ}$ and $26^{\circ} \mathrm{S}$. In: CONGR. GEOL. ARGENT., 10. Tucumán, 1987. Actas... Tucumán, Asoc. Geol. Argent. v. 4, p. 355-357.

GRISSOM, G.C.; DEBARI, S.M.; PAGE, S.P.; PAGE, R.F.; VILLAR, L.M.; COLEMAN, R.G.; RAMÍREZ, M.V. 1991. The deep crust of an early Paleozoic are; The Sierra de Fiambala, Northwestern Argentina. In: HARMON, R.S. \& RAPELA, C.W. eds. Andean magmatism and its tectonic setting. Geol. Soc. Am. Spec. Paper, 265:189-200.

HARTNADY, C.J.H. 1991. About turn for supercontinents. Nature, 352:476-478.

HOFFMAN, P.F. 1988. United plates of America, the birth of a craton: Early Proterozoic assembly and growth of Laurentia. Ann. Rev. Earth Planet. Sci., 16:543-603.

HOFFMAN, P.F. 1991. Did the breakout of Laurentia turn Gondwanaland inside-out? Science, 252:1409-1412.

HOFFMAN, P.F. 1992. Global Grenvillian kinematics and fusión of the Neoproterozoic supercontinent Rodinia. Canadá, Geol. Soc. Can. p. 17-49. (Progr. with Abstr.).

JEZEK, P.; WILLNER, A.P; ACEÑOLAZA, F.G ; MILLER, H. 1985. The Puncoviscana trough - a large basin of Late Precambrian to Early Cambrian age on the Pacific edge of the Brazilian shield. Geol. Rundsch., 74(3):573-584. 
KAZ'MIN, V.G. 1988. Tectonic development of the Mozambique zone: from accretion Geotectonics, 22:213-219.

LEHMANN, B. 1978. A Precambrian core sample from the Altiplano Boliviano. Geol. Rundsch., 67(1):270-278

LEVERATTO, M.A. 1968. Geología de la zona al oeste de Ullún-Zonda, borde oriental de la Precordillera de San Juan, eruptividad subvolcánica y estructura. Asoc. Geol Argent. Rev., 23(2): 129-157.

LINARES, E.; CAGNONI, M.; CAMPO, M.; OSTERA, H. 1985. Geocronología de las rocas metamórficas y eruptivas de la provincia del Neuquén y del noroeste de la provincia de Río Negro, República Aroentina. Rev Comunicaciones, 35:129-136.

LINARES, E.; CAGNONI, M.C.; CAMPO, M.; OSTERA, H.A. 1988 Geochronology of metamorphic and eruptiva rocks of southeastem Neusquén and northwestern Río Negro Provinces, Argentine Republic. J. South Am. Earth Sci., 1(1):53-61.

LITHERLAND, M.; KLINCK, B.A.; O'CONNOR, E.A.; PUFIELD P.E.J. 1985. Andean-trending mobile belts in the Brazilian shield. Nature, 314:345-348.

LITHERLAND, M.; ANNELLS, R.N.; DARNYSHIRE, D.P.F.; FLETCHER, C.J.N.; HAWKINS, M.P.; KLINCK, B.A.; MITCHELL, W.I. CONNOR, E.A; PITFIELD, P.E.J; POWER, P; WEBB, B.C. 1989. The Proterozoic of Eastern Bolivia and its relationship to the Andean Mobile Belt. Precambrian Res., 43:157-174.

LOSKE, W.P. 1992. Sedimentologie, herkunft und geotektonische entwicklung palaozoischer gesteine der Prakordillere WestArgentiniens. Münchner Geol. Hefte, 7:1-143.

McDONOUGH, M.R.; RAMOS, V.A.; ISACHSEN, CE.; BOWRING S.A.; VUJOVICH, G.I. 1993. Nuevas edades de circones del basamento de la Sierra de Pie de Palo, Sierras Pampeanas Occidentales de San Juan: sus implicancias para los modelos del supercontinente proterozoico de Rodinia. In: CONGR. GEOL. ARGENT., 12. Mendoza, 1993. Actas... Mendoza, Soc. Geol. Argent. v. 3, p. 340-342.

MOORE, G.W. 1992. Tectonic assembly of South America. Episodes, $15 \cdot 204-206$

MOORES, E.M. 1991. Southwest U.S. - East Antarctic (SWEAT) connection: a hypothesis. Geology, 19:425-428.

OMARINI, R.H. 1983. Caracterización Litológfca, Diferenciación y Génesis de la Formación Puncoviscana entre el Valle de Lerma y la Faja Eruptova de la Puna. Salta. 202 p. (Tesis Doctoral, Universidad Nacional Salta).

PALMA, M.A.; PARICA, P.; RAMOS, V.A. 1986. El Granito Archibarca: su edad y significativo tectónico. Asoc. Geol. Argent. Rev., 41(34):414-419.

RAMOS, V.A. 1988. Tectonics of the Late Proterozoic - Early Paleozoic: a collisional history of Southern South America. Episodes, 11(3): 168174.

RAMOS, V.A. \& VUJOVICH, G.I. 1993. Laurentia-Gondwana connetion: A Southamerican perspective. Geol. Soc. Am. Ann. Meet., Abstr. with Programs A-232

RAMOS, V.A.; JORDÁN, T.E.; ALLMENDINGER, R.W.; KAY, S.M.; CORTES, J.M.; PALMA, M.A. 1984. Chilenia: un terreno alóctono en la evolución paleozoica de los Andes Centrales. In: CONGR GEOL. ARGENT., 9. Bariloche, 1984. Actas... Bariloche, Soc. Geol. Argent. v. 2, p. 84-106.

RAMOS, VA.; JORDAN, T.E.; ALLMENDINGER, R.W.; MPODOZIS, C.; KAY, S.; CORTES, J.M.; PALMA, M.A. 1986. Paleozoic terranes of the Central Argentine-Chilean Andes. Tectonics, 5:855-880.

RAMOS, VA.; VUJOVICH, G.I; KAY, S.M.; McDONOUGH, M.R. 1993. La Orogénesis de Grenville en las Sierras Pampeanas
Occidentales: Pie de Palo, un sistema de aeración de arcos islándicos. In: CONGR. GEOL. ARGENT., 12. Mendoza, 1993. Actas... Mendoza, Soc. Geol. Argent. v. 3, p. 343-357.

RANKIN, D.W.; DRAKE, A.A., Jr.; GLOVER, L., III; GOLDSMITH, R.; HALL, L.M.; MURRAY, D.P.; RATCLIFFE, N.M.; READ, J.F.; SECOR, D.T.; STANLEY, R.S. 1989. Pre-orogenic terranes. In: HATCHER, R.D. Jr.; THOMAS, W.A.; VIELE, G.W. eds. The Geology of North America. The Appalachian-Ouachita Orogen in the United States. Geol. Soc. Am., f-2:7-100.

RÁPELA, C.W.; PANKHURST, R.J.; BONALUMI, A.A. 1991. Edad y

feoquímica del pórfido granítico de Oncán, Sierra Norte de Córdoba,

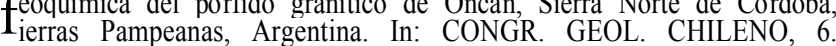
Santiago, 1991. Resúmenes Expandidos... Santiago, Servicio Nacional de Geología y de Minería de Chile, v. 1, p. 19-22.

RÁPELA, C.W.; COIRA, B.; TOSELLI, A.; SAAVEDRA, J. 1992. E magmatismo del Paleozoico Inferior en el Sudoeste de Gondwana. In: GUTIERREZ MARCO, J.; SAAVEDRA, J.; RÁBANO, L; SCHON, C.; MILER, H. eds. Paleozoico Inferior de íbero-América. Extremadura, Universidad de Extremadura, p. 21-68.

SCHON, C. \& MILLER, H. 1990. The evolution of the lower paleozoic trondhiemite/granite suites SW of Cachi, NW-Argentina. In: LAUBACHER, G.; OLIVIER, R.A.; VATIN-PERIGNpN, N. eds. Symp. Intern. Geodinamique Andine. Grenoble, Editions l'ORSTOM. p. 363-366.

SPALLETTI, L · CINGOLANI, C.A · VÁRELA, R · CURDA, A.J. 1989 Sediment gravity flow deposits of an Ordovician deep-sea fan system (westem Precordillera, Argentina). Sediment. Geol., 61:287-301.

STERN, R.J.; SULTAN, M.; GAMAL ABDEL-SALAM, M. 1992. Comment and Reply on "Pacific margins of Laurentia and East Antartica-Australia as a conjúgate rift pair: evidence and implications for en Eocambrian supercontinent". Geology, 20(2):190-191.

SUAREZ SORUCO, R. \& LOPEZ PAULSEN, O. 1992. En Guía de Campo: Ciclo Tacsariano. In: CONF. INTERN. CUENCAS FANEROZOICAS DEL GONDWANA OCCIDENTAL, 1. La Paz, 1992. Boletín. La Paz, Am. Ass. Petrol Geol - YPFB. p. 23-24.

UNRUG, R. 1992. The supercontinent cycle and Gondwanalan assembly: component cratons and the timing of suturing events. $/$. Geodynamics, 16(4):215-240.

VUJOVICH, G.I. 1992. Anfibolitas y metabasitas de la loma de Las Chacras, Sierra de La Herta, provincia de San Juan. In: BRODTKORB, M.K. \& SCHALAMUK, I. eds. J. Miner. Petrol. y Metalog. Rocas Ultrabás., 2:491-500.

VUJOVICH, G.1.1993. Arcos magmáticos islándicos en el sector occidental del terreno Pampeano. In: CONGR. GEOL. ARGENT., 12. Mendoza, 1993. Actas... Mendoza, Soc. Geol. Argent. v. 4, p. 17-22.

VUJOVICH, G.; RAMOS, VA.; MARIN, G.; MILLER, H. 1991 Proterozoic metavolcanics from western Sierras Pampeanas Terrane, Argentine. In: INT. CIRCUMPACIFIC TERRANE CONF., 4. Santiago, 1991. Res. Expand. Comunicaciones, 42:238-239.

VUJOVICH G.; MILLER, H.; RAMOS, V.A. 1992. Proterozoic metavolcanics from western Sierras Pampeanas Terrane, Argentine. J. South Am. Earth Sci., 7(3-4):309-323.

MANUSCRITO A790

Recebido em 13 de setembro de 1993

Revisáo do autor em 17 de dezembro de 1993

Revisáo aceita em 31 de marco de 1994 